“ (C) 2018 IEEE. Personal use of this material is permitted. Permission from IEEE must be obtained for all other uses, in any current or future media, including

reprinting/republishing this material for advertising or promotional purposes, creating new collective works, for resale or redistribution to servers or lists, or reuse of any copyrighted component of this work in other works." 


\section{Non-Contact Doppler Radar Based Prediction of Nocturnal Body Orientations Using Deep Neural Network for Chronic Heart Failure Patients}

\author{
Vinh Phuc Tran \\ Centre for Health Technologies \\ Faculty of Engineering \& Information Technology \\ University of Technology, Sydney (UTS) \\ Sydney, NSW, Australia \\ Vinh.P.Tran-1@student.uts.edu.au
}

\author{
Adel Ali Al-Jumaily \\ Centre for Health Technologies \\ Faculty of Engineering \& Information Technology \\ University of Technology, Sydney (UTS) \\ Sydney, NSW, Australia \\ Adel.Al-Jumaily@uts.edu.au
}

\begin{abstract}
Sleep is crucial in our daily life as it play a key role in our physical and mental health. It is important to monitor the sleep body orientations and movements due to its relationships to particular diseases, e.g., obstructive sleep apnea, insomnia, or periodic limb movement disorder. Analyzing sleep body orientations also helps in determining sleep quality and irregular sleeping patterns. However, current non-invasive sleep body orientations monitoring technology is not well suited for longterm continuous monitoring due to its restrictions in mobility and comfort. This paper proposed a system that applies a feature extraction process utilizing wavelet packet decomposition to extract predictable sets of features that describe the non-contact Doppler radar signatures caused by the body orientations. A database consisting of $\mathbf{2 4}$ chronic heart failure patients with New York Heart Association heart failure classification Class II \& III; whose underwent full polysomnography analysis for diagnosis of sleep apnea, disordered sleep, or both, is selected for the training, validation and test of the non-contact body orientations prediction. The patients' dataset is partitioned randomly into the ratio of $50 \%$ for training, $15 \%$ for validation and $35 \%$ for test. Across the test dataset with total sleep duration of 65 hours, the body orientations prediction accuracy achieved a coherence high correct classification rate of $99.2 \%$ for 5 classes of 'Prone', 'Upright', 'Supine', 'Right' and 'Left' body orientations. The misclassification rate also cohered with a negligible rate of $0.8 \%$. A potential application would be non-contact continuous monitoring of nocturnal body orientations in the home.
\end{abstract}

Keywords-Non-contact Doppler radar; Non-contact body orientations; Non-contact sleep monitoring;

\section{INTRODUCTION}

Obstructive sleep apnea (OSA) is a common and potentially lethal sleep disorder affecting at least $4 \%$ of adult males and $2 \%$ of adult females world-wide [1]. Statistics published in 2013 reported that the prevalence of OSA had increased to $10 \%-17 \%$ for adult males and 3\%-9\% for adult females in the United States of America (USA) [2].

OSA is the cessation of airflow due to the collapse of the upper airway during sleep and can occur at any age, from infancy to old age. Evidences have indicated that OSA is associated with ischemic heart disease, increased prevalence of stroke, coronary artery disease, atrial fibrillation, chronic heart failure (CHF) and cardiac sudden death [3].

The importance of sleep monitoring is not just limited to physiological vital signs, apnea-hypopnea index (AHI), and sleep/wake patterns, it is also important to monitor the sleep body orientations and movements due to its relationships to particular diseases, e.g., OSA, insomnia (sleeplessness), or periodic limb movement disorder (PLMD). Analyzing sleep body orientations also helps in determining sleep quality and irregular sleeping patterns.

Non-contact biosensor such as microwave Doppler radar for physiological vital signs monitoring has been known since 1970's. Enormous literatures have been published regarding non-contact assessments of respiratory and heart rates. However, the non-contact prediction of nocturnal body orientations using microwave Doppler radar in the complexity of sleep environment has not yet been reported. It is also important to emphasize that the majority of current reported achievements for non-contact microwave Doppler radar, single or multi-radar systems, are based on 'stationary' and 'directfacing' subject measurements, which is not a real life scenario for the complexity of sleep environment.

This paper proposed a novel non-contact Doppler radar based prediction of nocturnal body orientations using deep neural network for CHF patients. In addition, this paper employ a feature extraction process utilizing wavelet packet decomposition (WPD) to extract predictable sets of features that describe the non-contact Doppler radar signatures caused by the body orientations.

This paper is structured as follows: section II describes the non-contact biosensor that tracks a person's movement while sleeping using a radiofrequency motion sensor. Based on these signals, the features are extracted via a wavelets extraction process as explained in section III. The extracted features are then trained through a deep neural network architecture as proposed in section IV and V. Section VI reports the results of the body orientations prediction using deep neural network as compared with the gold-standard PSG recordings from a set of 
24 CHF patients. Finally, section VII concludes the work presented in this paper.

\section{MicrowaVe DopPler RADAR \& PATIENTS DATABASE}

\section{A. SleepMiner ${ }^{T M}$ Biosensor}

The SleepMinder ${ }^{\mathrm{TM}}$ (SM) is a ResMed Ltd.'s patented (WO/2013/177621) [4] microwave radar designed for the convenient measurement of sleep and breathing in the home. $\mathrm{SM}$ is a dual pulse-Doppler radar designed to transmit two short pulses of radio frequency energy at $5.8 \mathrm{GHz}$ and capable of measuring movements at a distance between $0.5-3.0$ meters. SM also employs quadrature detection techniques, which leads to two estimates of the movement signals, called I and Q channels. The outputs I and Q channels are internally filtered by active analogue filters at $1.6 \mathrm{~Hz}$ and sampled at 64 $\mathrm{Hz}$. The $64 \mathrm{~Hz}$ samples are then averaged over 4 samples, producing two $16 \mathrm{~Hz}$ channels and saved to the SM memory card in a proprietary binary format.

An illustration of the SM setup in a sleep laboratory or at home is shown in Fig. 1.

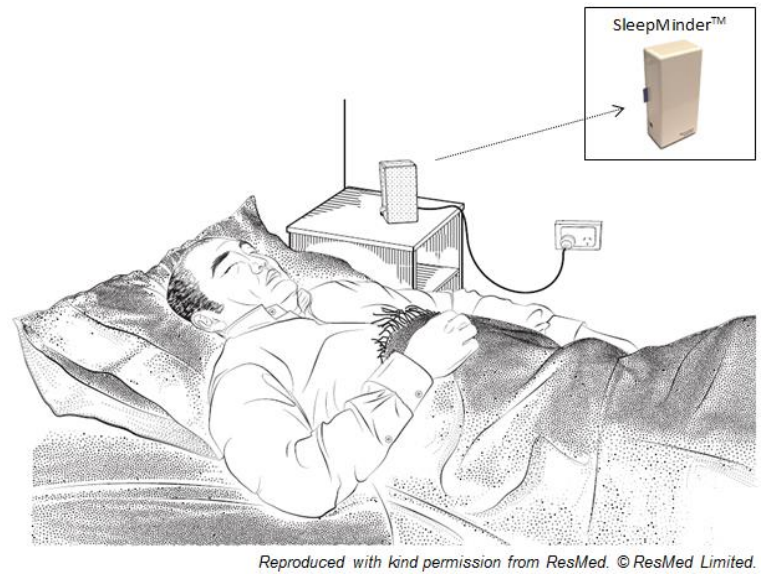

Fig. 1. SleepMinder ${ }^{\mathrm{TM}}$ setup in a sleep laboratory or at home

\section{B. Patients Database}

A database consisting of 24 chronic heart failure (CHF) patients with New York Heart Association (NYHA) heart failure classification Class II \& III is selected for the training, validation and independent test of the body orientations prediction using deep neural network. The patients groups are of 2 females and 22 males, who were sequentially admitted in the Royal Brompton Centre for Sleep, London, United Kingdom, for the diagnosis of sleep apnea, disordered sleep, or both. The patients' mean age is 69.05 years, with mean body weight of $82.69 \mathrm{~kg}$, mean body height of $1.72 \mathrm{~m}$, mean BMI of 28.01, and mean recorded sleep duration of 7.76 hours. The consented patients underwent full polysomnography (PSG) analysis with manually scored by sleep experts.

The SM was installed in the sleep laboratory and its signals were recorded simultaneously with the PSG signals. The SM was positioned facing the patient in line with chest, at a distance of 0.5 meter, and an elevation of 0.5 meter from the edge of the bed.

\section{FeAture EXtraction Using WAVELET PACKET DECOMPOSITION}

\section{A. Wavelets Extraction Process}

The process of wavelets extraction from the raw I \& Q channels is proposed in Fig. 2.

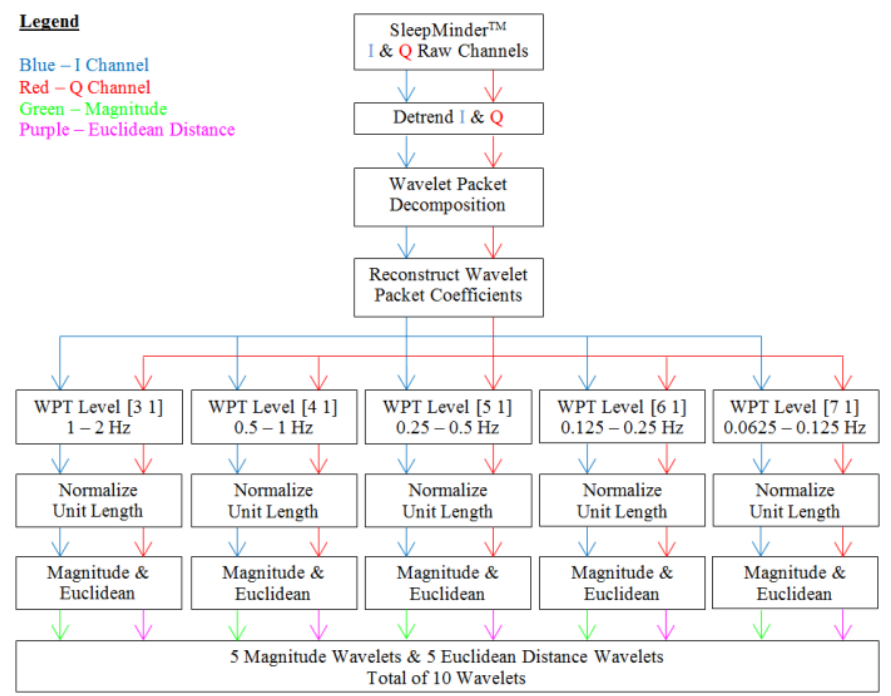

Fig. 2. Wavelets Extraction Process

Prior applying wavelet packet decomposition (WPD), the signal is filtered where DC offsets were removed by subtracting the mean from the raw I \& Q channels. The detrended I \& Q channels are then decomposed to $7^{\text {th }}$ level with both 'approximations' and 'details' coefficients decomposed. The mother wavelet for WPD is Symlet wavelet with $4^{\text {th }}$ order filter. The reason for choosing the Symlet wavelet is for the performance and accuracy as detailed in the authors' previous work [5].

The proposed categories of the frequencies of interest are:

- Slow body movements - Doppler signatures are reflected at frequencies between greater than $0 \mathrm{~Hz}$ and $0.25 \mathrm{~Hz}$.

- $\quad$ Respiratory chest movements - Doppler signatures are reflected at frequencies between $0.25 \mathrm{~Hz}$ and $0.5 \mathrm{~Hz}$, correspond to $7.5-30$ breaths per minute.

- Heart chest movements - Doppler signatures are reflected at frequencies between $0.5 \mathrm{~Hz}$ and $1.0 \mathrm{~Hz}$, correspond to $30-120$ heart beats per minute.

- Fast body movements - Doppler signatures are reflected at frequencies between $1.0 \mathrm{~Hz}$ and $2.0 \mathrm{~Hz}$.

Therefore, only the 'details' coefficients are of interest. The decomposed 'details' coefficients are then reconstructed at the wavelet packet tree (WPT) levels as indicated in Fig. 2. The reconstructed wavelets are then normalized to unit length.

To combine the normalized I \& Q wavelets, the magnitude of I \& Q and the Euclidean distance of I \& Q are calculated for 
each of the normalized I \& Q wavelets. This produced a total of 10 unique wavelets, which contains 5 unique magnitude wavelets and 5 unique Euclidean distance wavelets.

\section{B. Features Extraction Process}

The proposed features extraction process extracted features as per wavelet, with a fixed window-width of 60 seconds (2 epochs) and a sliding window-width of 30 seconds (1 epoch) for the entire length of the wavelet. Two types of features are extracted as per window, one is time-domain statistical features and the other is frequency-domain features.

Table I indicate the 11 time-domain statistical features as per window, where ' $x$ ' is the selected signal at the selected window. The statistical methods for obtaining the features are mean, variance, median-absolute-deviation, standard deviation, natural logarithm of mean and geometric mean.

TABLE I

TIME-DOMAIN STATISTICAL FEATURES

\begin{tabular}{|c|c|c|c|}
\hline \hline $\operatorname{mean}(\mathrm{x})$ & $\operatorname{mean}\left(\mathrm{x}^{2}\right)$ & $\ln (\operatorname{mean}(\mathrm{x}))$ & $\ln \left(\operatorname{mean}\left(\mathrm{x}^{2}\right)\right)$ \\
\hline $\operatorname{var}(\mathrm{x})$ & $\operatorname{var}\left(\mathrm{x}^{2}\right)$ & $\operatorname{std}(\mathrm{x})$ & $\operatorname{std}\left(\mathrm{x}^{2}\right)$ \\
\hline $\operatorname{mad}(\mathrm{x})$ & $\operatorname{mad}\left(\mathrm{x}^{2}\right)$ & $\operatorname{geomean}\left(\mathrm{x}^{2}\right)$ & \\
\hline \hline
\end{tabular}

Table II indicate the methods for obtaining the frequencydomain features as per window. Fast Fourier transform (FFT) is performed on the selected window. The absolute magnitudes of the frequency components and the derivative of the absolute magnitudes are also calculated. The sum of the absolute magnitudes and the sum of the derivative of the absolute magnitudes are obtained as features. The power spectral density (PSD) is also performed on the selected window. The PSD magnitudes and the derivative of the PSD magnitudes are also calculated. The sum of the PSD magnitudes and the sum of the derivative of the PSD magnitudes are obtained as features.

TABLE II

FREQUENCY-DOMAIN FEATURES

\begin{tabular}{|c|c|}
\hline \hline sum(abs_mag) & sum(psd) \\
\hline sum(diff_abs_mag) & sum(diff_psd) \\
\hline \hline
\end{tabular}

The proposed features extraction process produced a total of 150 features, which is the product of 10 wavelets and the sum of 11 time-domain statistical features and 4 frequencydomain features. The total 150 features are then re-scaled to the range of -1 to 1 .

\section{Body ORIENTATIONS PREDICTION DEEP NEURAL NETWORK ARCHITECTURE}

The body orientations prediction deep neural network architecture is proposed in Fig. 3. The deep neural network architecture is implemented using fully connected multilayer perceptron (MLP) with bias of ' 1 '. The deep neural network contains 5 layers, in which 4 layers are hidden layers and 1 output layer. The output layer contains 5 binary classes of 'Prone', 'Upright', 'Supine', 'Right' and 'Left' body orientations.

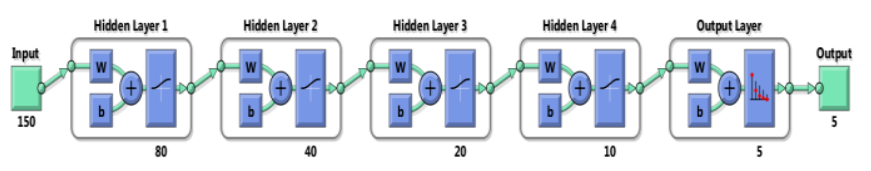

Fig. 3. Body Orientations Prediction Deep Neural Network Architecture

The summary of the deep neural network architecture characteristics is shown in Table III. The number of neurons per layer is selected as half of the previous layer's neurons with the goal of reducing the non-linear high-dimensional features when propagate through each layer. The total neurons of the hidden layers are 150.

TABLE III

DEEP NEURAL NETWORK ARCHITECTURE CHARACTERISTICS

\begin{tabular}{|c|c|c|c|c|c|}
\hline \hline & $\begin{array}{c}\text { Hidden } \\
\text { Layer 1 }\end{array}$ & $\begin{array}{c}\text { Hidden } \\
\text { Layer 2 }\end{array}$ & $\begin{array}{c}\text { Hidden } \\
\text { Layer 3 }\end{array}$ & $\begin{array}{c}\text { Hidden } \\
\text { Layer 4 }\end{array}$ & $\begin{array}{c}\text { Output } \\
\text { Layer }\end{array}$ \\
\hline $\begin{array}{c}\text { Number of } \\
\text { Neuron }\end{array}$ & 80 & 40 & 20 & 10 & 5 \\
\hline $\begin{array}{c}\text { Activation } \\
\text { Function }\end{array}$ & $\begin{array}{c}\text { Logistic } \\
\text { Sigmoid }\end{array}$ & $\begin{array}{c}\text { Logistic } \\
\text { Sigmoid }\end{array}$ & $\begin{array}{c}\text { Logistic } \\
\text { Sigmoid }\end{array}$ & $\begin{array}{c}\text { Logistic } \\
\text { Sigmoid }\end{array}$ & Softmax \\
\hline \hline
\end{tabular}

\section{Dataset Partition \& DeEp Neural Network Training CHARACTERISTICS}

The total $24 \mathrm{CHF}$ patients dataset stated in section II is partitioned randomly into 3 datasets of 'Training', 'Validation' and independent 'Test'. The dataset partition categories are summarized in Table IV. The training characteristics of the deep neural network are summarized in Table V.

TABLE IV

DATASET PARTITION CATEGORIES

\begin{tabular}{|c|c|c|c|c|c|}
\hline \hline \multirow{2}{*}{} & $\begin{array}{c}\text { Dataset } \\
(\mathbf{1 0 0 \% )}\end{array}$ & $\begin{array}{c}\text { Training } \\
\mathbf{( 5 0 \% )}\end{array}$ & $\begin{array}{c}\text { Validation } \\
(\mathbf{1 5 \% )}\end{array}$ & $\begin{array}{c}\text { Test } \\
\mathbf{( 3 5 \% )}\end{array}$ \\
\hline $\begin{array}{c}\text { Sample } \\
\text { Count }\end{array}$ & Total & 10698240 & 5349120 & 1604736 & 3744384 \\
\cline { 2 - 6 } & Prone & 95520 & 47709 & 14345 & 33466 \\
\cline { 2 - 6 } & Upright & 566387 & 283456 & 84402 & 198529 \\
\cline { 2 - 6 } & Supine & 2412959 & 1206070 & 362890 & 843999 \\
\cline { 2 - 6 } & Right & 2381283 & 1190890 & 357192 & 833201 \\
\cline { 2 - 6 } & Left & 5242091 & 2620995 & 785907 & 1835189 \\
\hline $\begin{array}{c}\text { Sleep } \\
\text { Hour }\end{array}$ & Total & 185.7 & 92.9 & 27.9 & 65.0 \\
\cline { 2 - 6 } & Prone & 1.7 & 0.8 & 0.2 & 0.6 \\
\cline { 2 - 6 } & Upright & 9.8 & 4.9 & 1.5 & 3.4 \\
\cline { 2 - 6 } & Supine & 41.9 & 20.9 & 6.3 & 14.7 \\
\cline { 2 - 6 } & Right & 41.3 & 20.7 & 6.2 & 14.5 \\
\cline { 2 - 6 } & Left & 91.0 & 45.5 & 13.6 & 31.9 \\
\hline $\begin{array}{c}\text { \% of } \\
\text { Total }\end{array}$ & Prone & 0.9 & 0.9 & 0.9 & 0.9 \\
\cline { 2 - 6 } Sample & Upright & 5.3 & 5.3 & 5.3 & 5.3 \\
\cline { 2 - 6 } & Supine & 22.5 & 22.5 & 22.5 & 22.5 \\
\cline { 2 - 6 } & Right & 22.3 & 22.3 & 22.3 & 22.3 \\
\cline { 2 - 6 } & Left & 49.0 & 49.0 & 49.0 & 49.0 \\
\hline \hline
\end{tabular}

TABLE V

DEEP NEURAL NETWORK TRAINING CHARACTERISTICS

\begin{tabular}{|c|c|c|c|}
\hline $\begin{array}{c}\text { Derivative } \\
\text { Function }\end{array}$ & $\begin{array}{c}\text { Network } \\
\text { Initialization }\end{array}$ & $\begin{array}{c}\text { Performance } \\
\text { Function }\end{array}$ & $\begin{array}{l}\text { Training } \\
\text { Function }\end{array}$ \\
\hline $\begin{array}{c}\text { Static } \\
\text { derivative } \\
\text { function } \\
\text { (staticderiv- } \\
\text { chain rule) }\end{array}$ & $\begin{array}{c}\text { Layer-by- } \\
\text { layer: } \\
\text { Nguyen- } \\
\text { Widrow } \\
\text { initialization } \\
\text { function } \\
\text { (initnw) }\end{array}$ & $\begin{array}{l}\text { Cross Entropy } \\
\text { Regularization: } 0.001 \\
\text { Normalization: none }\end{array}$ & $\begin{array}{c}\text { Scaled } \\
\text { Conjugate } \\
\text { Gradient } \\
\\
\sigma=0.005 \\
\lambda=0.0005\end{array}$ \\
\hline
\end{tabular}




\section{RESULTS \& DISCUSSIONS}

The deep neural network is trained with a maximum validation failed criteria of 6 epochs, i.e., the training will be stopped after a maximum of 6 consecutive epochs of 'Validation' errors increases when compared to 'Training' errors. The network is stopped at 32941 epochs according to the maximum validation failed criteria. The epoch just before the first validation failed criteria is at 32935 , and this is referred to as the 'best' epoch. The network weights are obtained at the 'best' epoch.

The training performance of the deep neural network is illustrated in Fig. 4. As shown in Fig. 4, all errors of 'Training', 'Validation' and independent 'Test' decreases and approaches zero as training progresses. This indicates that the input features are predictable features, the dataset size is adequate to prevent under-fitting, the network is well regularized to prevent over-fitting and the network had successfully learned the features characteristics. It is important to emphasize that the independent 'Test' dataset is used for monitoring purpose only and does not participate in training performance criteria.
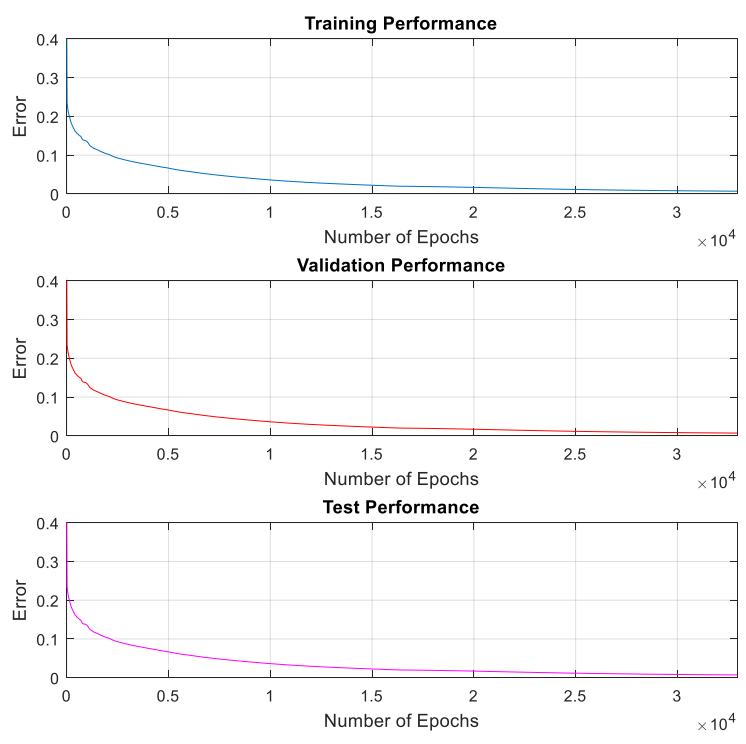

Fig. 4. Deep Neural Network Training Performance Plot

The results of the body orientations prediction is presented in Fig. 5 as confusion matrix. As shown in Fig. 5, 'Training', 'Validation' and independent 'Test' accuracy achieved a coherence high correct classification rate of $99.2 \%$ for 5 binary classes of body orientations. The misclassification rate also cohered with a negligible rate of $0.8 \%$. Figure 5 class labels of ' 1 ' correspond to 'Prone', '2' correspond to 'Upright', '3' correspond to 'Supine', '4' correspond to 'Right' and '5' correspond to 'Left' body orientations.

Figure 6 present the body orientations prediction for the independent 'Test' dataset. As shown in Fig. 6, the non-contact prediction of nocturnal body orientations using deep neural network accurately predicted the correct body orientations as compared to the reference body orientations recorded by the PSG system with an accuracy of $99.2 \%$. This result had proven the feasibility of non-contact Doppler radar based prediction of nocturnal body orientations using deep neural network for $\mathrm{CHF}$ patients in the complexity of sleep environment.
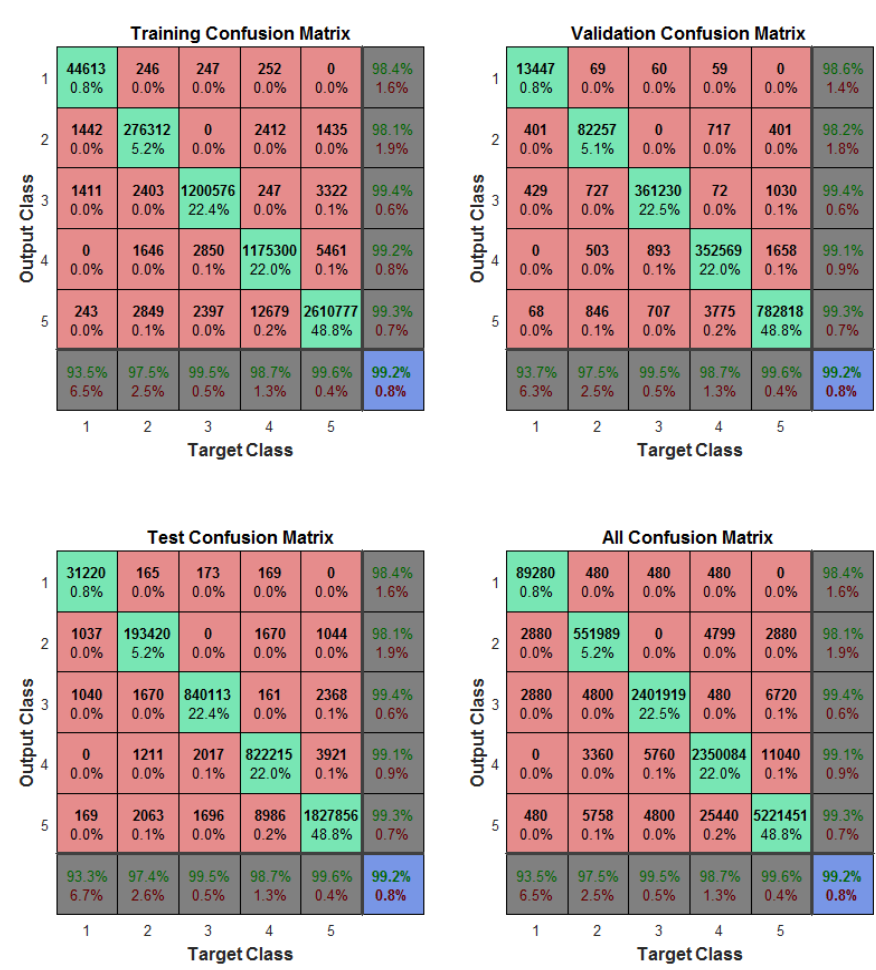

Fig. 5. Deep Neural Network Confusion Matrix
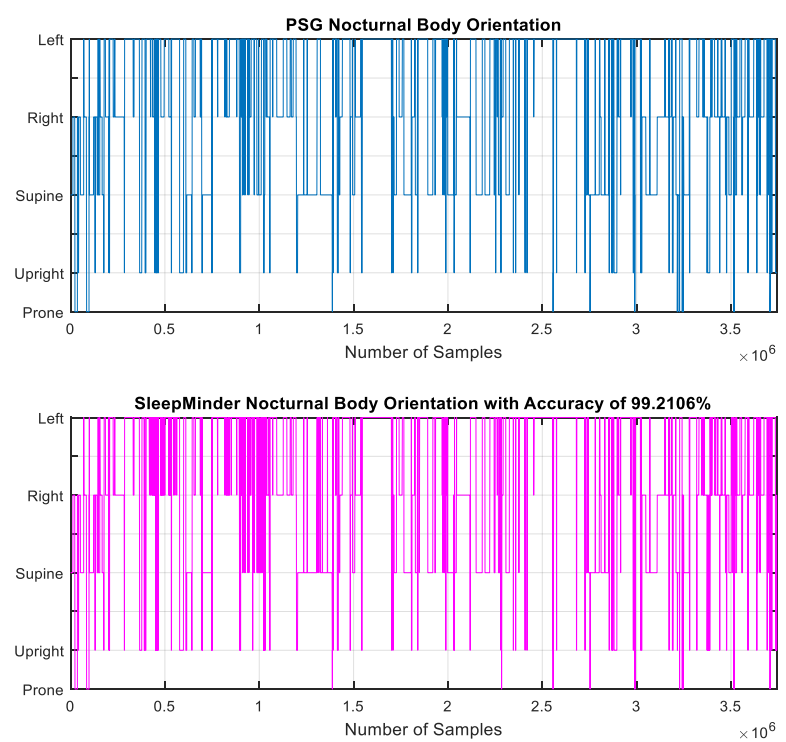

Fig. 6. Independent 'Test' Dataset - Nocturnal Body Orientations

Figure 7 present the non-contact prediction of nocturnal body orientations for a CHF patient. As shown in Fig. 7, the non-contact body orientations prediction is exceptionally accurate compared to the reference body orientations recorded by the PSG system. 

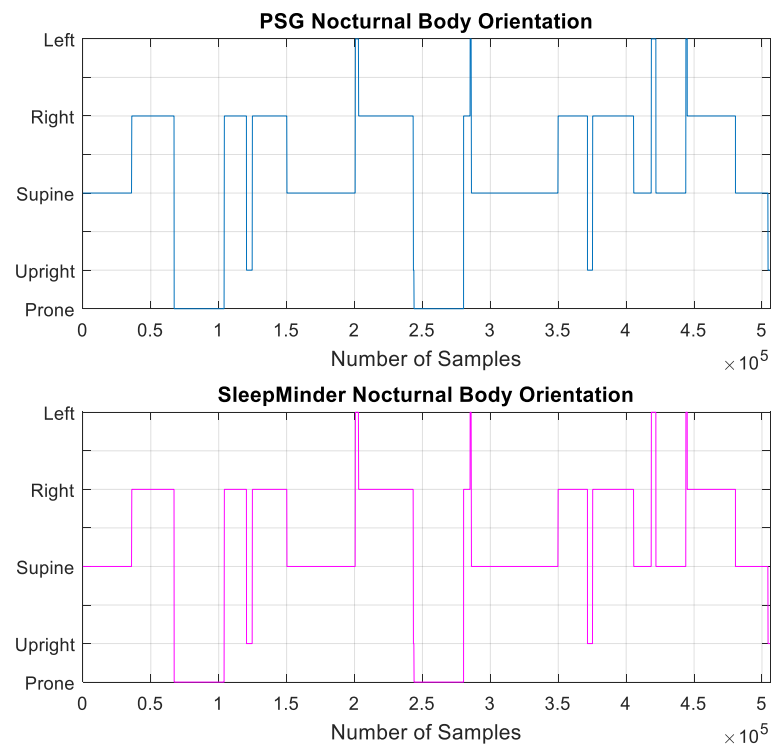

Fig. 7. Nocturnal Body Orientations for a CHF patient

Figure 8 is a time-series illustration of the features for a CHF patient. The total of 150 features is plotted against the samples of the patient's entire sleep duration to help visualizing the features corresponding to body orientations. However, the features does not correspond to meaningful phenomenon, therefore, it is only applicable for machine learning applications, such as, deep neural network in this research work and is incomprehensible to human logic.
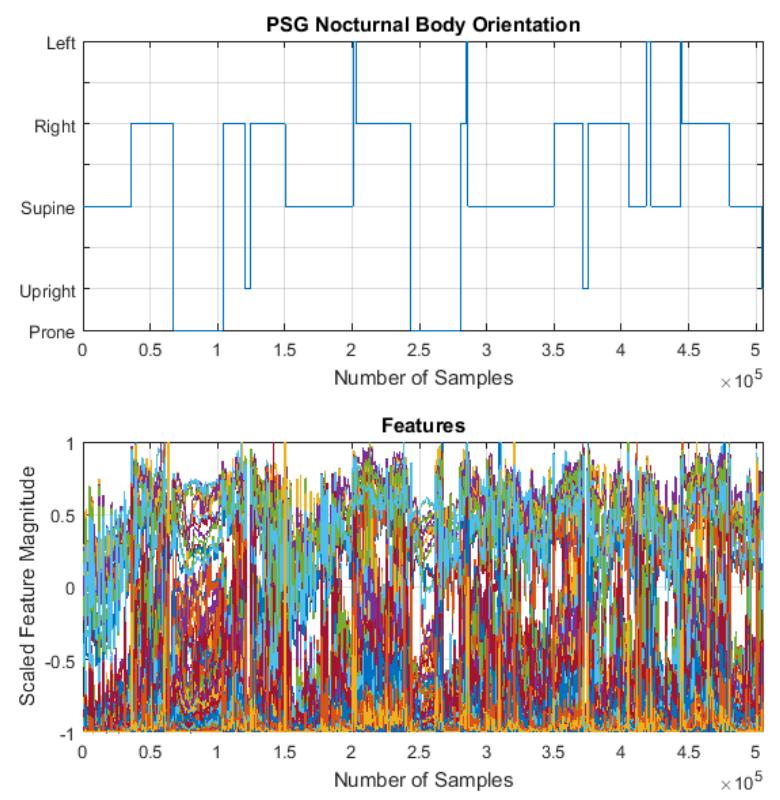

Fig. 8. Features Time-Series Illustration for a CHF Patient

\section{CONCLUSION}

This paper has demonstrated and proven the feasibility of non-contact Doppler radar based prediction of nocturnal body orientations using deep neural network for CHF patients in the complexity of sleep environment.

The achievement is at a high correct classification rate of 99.2\% for 5 classes of 'Prone', 'Upright', 'Supine', 'Right' and 'Left' body orientations. The misclassification rate is at a negligible rate of $0.8 \%$.

A potential application would be a non-contact system that continuously monitors the body orientations during sleep in the home. More importantly, the non-contact system eliminate the need for body-contact sensors, which in turn addresses the current non-invasive sleep body orientations monitoring technology restrictions, such as, limited mobility, irritations, distress and discomforts to the patient under monitoring.

\section{ACKNOWLEDGEMENT}

The authors would like to acknowledge the support of the Australian Government Research Training Program Scholarship. The authors also would like to acknowledge the support of ResMed Ltd. in providing the SleepMinder ${ }^{\mathrm{TM}}$ and patients' database for this research.

\section{DISCLOSURE STATEMENT}

The authors have no relevant conflicts of interest to disclose.

\section{REFERENCES}

[1] T. Young, M. Palta, J. Dempsey, J. Skatrud, S. Webber, and S. Badr, "The Occurrence of Sleep-Disordered Breathing Among Middle-Aged Adults," The New England Journal of Medicine, vol. 328, no. 17, pp. 1230-1235, 1993.

[2] P. E. Peppard, T. Young, J. H. Barnet, M. Palta, E. W. Hagen, and K. M. Hla, "Increased Prevalence of Sleep-Disordered Breathing in Adults," American Journal of Epidemiology, vol. 177, no. 9, pp. 10061014, 14 April, 2013, 2013.

[3] R. S. T. Leung, and T. D. Bradley, "Sleep Apnea and Cardiovascular Disease," American Journal of Respiratory and Critical Care Medicine, vol. 164, no. 12, pp. 2147-2165, 2001.

[4] K. H. Schindhelm, S. P. Farrugia, M. W. Colefax, F. Javed, R Khushaba, C. Heneghan, C. Philip De, A. Zaffaroni, N. Fox, P. Celka, E. O'Hare, and S. J. Redmond, (WO2013177621) Method and Apparatus for Monitoring Cardio-Pulmonary Health, World Intellectual Property Organization, 2013.

[5] V. P. Tran, and A. A. Al-Jumaily, "Non-Contact Dual Pulse Doppler System based Respiratory and Heart Rates Estimation for CHF Patients," in 37th Annual International Conference of the IEEE Engineering in Medicine and Biology Society, MiCo - Milano Conference Center - Milan, Italy, 2015, pp. 4202-4205. 